\title{
Capítulo 7 Comunicación estratégica de la ciencia con YouTube: el papel del comunicador/científico
}

\author{
Andrea De Santis-Piras \\ Universidad Politécnica Salesiana, (Ecuador), Universitat Autónoma \\ de Barcelona (España) \\ Orcid: https://orcid.org/oooo-0oo2-7441-3794. \\ Raquel Victoria Jara Cobos \\ Universidad Politécnica Salesiana, (Ecuador) \\ Orcid: https://orcid.org/oooo-0oo3-4947-5309 \\ DOI: https://doi.org/ 10.16921/ciespal.14.9
}

\section{Resumen}

En la sociedad del conocimiento las personas necesitan de la ciencia para entender y adaptarse a un entorno en constante desarrollo y, en muchas partes del mundo, Internet es el medio preferido para acceder a la información científica. A pesar de la aún limitada participación de los científicos en las nuevas dinámicas comunicativas que involucran los medios y redes sociales, existe un consenso en el reconocer a YouTube como la plataforma de videos en línea más utilizada por esta comunidad. En el presente capítulo se analiza YouTube como medio de comunicación científica evidenciando sus características como herramienta para favorecer la difusión y comprensión de conocimientos científicos. La reflexión apunta hacía el rol que juegan los académicos e investigadores en este proceso y el uso que hacen de YouTube para su comunicación, en particular de aquellos que investigan en el ámbito de la Publicidad y las Relaciones Públicas. Su presencia y participación es todavía escasa, se 
limita a iniciativas aisladas, y todavía son pocos los que se exponen fuera de los cánones y plataformas tradicionales de comunicación científica, perdiendo asíla oportunidad de incrementar la visibilidad e impacto de su labor. Se concluye que el uso de YouTube como herramienta estratégica de comunicación puede ser particularmente útil a las realidades asociativas e institucionales como grupos, core, centros e institutos de investigación.

Palabras clave: YouTube; comunicación científica; comunicación estratégica; medios sociales; presencia web.

\begin{abstract}
In the knowledge society, people need science to understand and adapt to a constantly developing environment and the Internet is the preferred medium for accessing scientific information in many parts of the world. Despite the still limited participation of scientists in the new communication dynamics involving media and social networks, there is a consensus in recognizing YouTube as the most widely used online video platform for this community. This chapter analyses YouTube as a media for scientific communication, highlighting its characteristics as a tool to promote the dissemination and understanding of scientific knowledge. The reflection points to the role that academics and researchers play in this process and the use they make of YouTube for their communication, particularly for researchers in the field of Advertising and Public Relations. Their presence and participation is still scarce, limited to isolated initiatives, and not many scientists choose to expose themselves outside the traditional canons and channels of scientific communication, thus losing the opportunity to increase the visibility and impact of their scientific work. In conclusion, the use of YouTube as a strategic communication tool can be particularly useful to associative and institutional realities such as research groups, core, centres and institutes.
\end{abstract}

Keywords: YouTube; science communication; strategic communication; social media; web presence

El 14, de febrero de 2020 YouTube cumplió sus primeros quince años con números de récord siendo la segunda plataforma web social con más usuarios activos después de Facebook alcanzando los 2.00o millones según el proveedor de datos Statista (2020), y el 79\% de la población mundial en Internet tiene su propia cuenta activa en 
YouTube (Datareportal, 2019). Funciona en más de 100 países con 80 idiomas distintos, sus usuarios visualizan 1.000 millones de horas de video cada día, concentrando el $37 \%$ de tráfico mundial de Internet móvil contra el 10\% que registran Facebook, Instagram y Whatsapp (Armstrong, 2019). Desde sus inicios, se ha caracterizado por la libertad y el alcance comunicativo que ofrece a los usuarios menos expertos en la elaboración de videos, revolucionando el concepto tradicional de canal televisivo transmisor de contenidos a una plataforma online donde todos pueden compartir información en formato audiovisual en diversas redes y medios de comunicación de la web (Biddinika et al., 2019). Sin duda, al revisar con más atención los números, se evidencia como la ciencia no se encuentra entre los tópicos más buscados en YouTube (Datareportal, 2019), dato preocupante si se considera que es el segundo sitio web más visitado al mundo después de Google.com (Alexa, 2020).

Sin embargo, la facilidad de acceso no garantiza la comprensión y correcta utilización de la información consumida, sobre todo cuando se trata de argumentos complicados o que requieren de mayor atención como la ciencia. En este capítulo, se analiza el rol que YouTube desempeña como medio de comunicación científica mediante una revisión de las principales contribuciones científicas evidenciado las características que esta plataforma ofrece a científicos, instituciones y público para comunicar sobre la ciencia y favorecer la difusión y comprensión de conocimientos científicos. Se ofrece también una contribución a la investigación sobre el uso de YouTube por parte de los científicos presentando los resultados obtenidos de una muestra de investigadores que se ocupan de Publicidad y Relaciones Públicas.

\section{Comunicación científica online}

Todos los grandes descubrimientos e inventos de la humanidad, para ser conocidos y aprovechados, deben ser comunicados. "Desde la antigüedad la difusión del conocimiento ha significado el éxito o la 
derrota de sociedades enteras, así como su sobrevivencia a lo largo de los siglos" (De Santis-Piras y Jara, 2019, p.131). Los responsables de la producción y gestión del conocimiento en la sociedad son llamados a divulgar sus resultados y hallazgos, y la web brinda la oportunidad de conectar los distintos sectores involucrados en el proceso haciendo posible, al mismo tiempo, la divulgación en públicos especializados y la apropiación del conocimiento por parte de los menos expertos (De Santis-Piras y Jara, 2019). Internet es el medio de comunicación más utilizado para acceder a la información científica en países como España (FECYT, 2018). Científicos, comunicadores y aficionados de todo el mundo han desarrollado habilidades y herramientas para difundir sus contenidos científicos con fines que van más allá de la educación del público (Francés y Peris, 2018; Laakso, Lindman, Shen, Nyman y Björk, 2017). La proliferación de plataformas de medios y redes sociales han revolucionado el entorno académico favoreciendo la aparición de nuevos formatos para la divulgación (Robinson-García, Repiso y Torres-Salinas, 2018) que pueden favorecer la adquisición de conocimientos científicos (Brossard, 2013).

La disponibilidad de contenido por sí misma no es suficiente y es necesario motivar los públicos a interesarse en la ciencia mediante contenidos y productos comunicacionales atractivos, más entretenidos y comprensibles para todo tipo de público. Bajo este argumento, los videos en línea son una herramienta estratégica para la comunicación científica, con cierto poder de influencia sobre las decisiones de compra y de comportamiento de los individuos (Oh, Baek y Ahn, 2017; Tseng y Huang, 2016), capaces de llegar a grandes audiencias. La visualización de un contenido permite la interacción con la información, hace visible lo que se imagina, materializando incluso conceptos abstractos: "Los investigadores empiezan a comprender que necesitan algo más que números; y los estudiosos del tema afirman que la necesidad técnica del hoy y el imperativo cognitivo del mañana es el uso de la imagen" (Díaz Pérez, de Moya Anegón y Carrillo-Calvet, 2018, p.5). 


\section{Uso del video online para la difusión de la ciencia}

El uso del formato en video aumenta la comprensión de la información que se presenta en formato escrito (Sonne, Andrews y Gentilin, 2013) facilitando la asimilación de cuestiones complicadas, como las que trata de explicar la ciencia. de Lara González y García-Avilés (2019) explican que los vídeos online sobre ciencia tienen un objetivo informativo, son de tipología muy variada, breves y de fácil comprensión, pero todavía no incluyen elementos de entretenimiento. Es por esto que cientificos, instituciones de educación, grupos y centros de investigación de distintos ámbitos consideran este tipo de recursos para dar a conocer sus actividades, difundir información relevante para la comunidad (Vázquez-Cano, 2013) y comercializar su trabajo (Allgier, 2020).

El vídeo en línea consiste en cualquier forma de contenido audiovisual que pueda verse a través de Internet, principalmente en plataformas de carga libre de contenido como YouTube, Youku, Hulu o Vimeo, plataformas para películas y series, vídeos producidos para el consumo en teléfonos móviles y tabletas; videoconferencias, videoblogs y otros formatos (Leon y Burke, 2018, p.2)

Un caso evidente, y entre los más estudiados, sobre cómo utilizar el video para la comunicación científica es el Journal of Visualized Experiments $(\mathrm{JoVE})^{4}$, primera vídeo-revista científica revisada por pares que difunde experimentos y protocolos científicos realizado en laboratorios de todo el mundo. JoVE es indexada por las bases de datos científicas de mayor impacto como Pubmed/MEDLINE, Web of Science y Scopus, y su factor de impacto en 2018 es 1,108. Cabe mencionar que la revista en cuestión es utilizada aproximadamente por mil instituciones de todo el mundo entre universidades, colegios, hospitales y compañías de biotecnología, y su objetivo principal consiste en diseminar los resultados de investigaciones científicas ofreciendo a científicos, educadores y estudiantes la posibilidad de visualizar los experimentos 
en detalle en lugar de leerlos en artículos de texto. Mediante el formato vídeo, JoVE incrementa la productividad de la investigación, favorece la reproducibilidad científica, el aprendizaje, y otorga mayor eficacia al proceso de comunicación de científicos, centros e instituciones (JoVE, 2020). Su contribución al campo de los estudios experimentales y al progreso científico en general es reconocida por la comunidad académica (Komada et al., 2009; Stern, 2013), pero se dirige a públicos especializados y no es de libre acceso en cuanto funciona con un sistema de pago por suscripción, aunque ofrece un período gratuito de prueba para nuevos usuarios.

Un ulterior ejemplo,es constituido por las TED Talks, charlas y conferencias de los congresos TED (Technology, Entertainment, Design) realizados por la organización sin fines de lucro estadounidense con el mismo nombre. Investigadores de varias disciplinas han analizado aspectos como las características psicológicas que producen las percepciones sobre investigación científica (Gheorghiu, Callan, y Skylark, 2020), la efectividad de este tipo de videos online para la comunicación médica como el cáncer (Verjovsky y Jurberg, 2019) Entertainment, Design (TED, el impacto de los videos TED en la comunicación científica en China (Pan, Yan y Hua, 2015) y su uso estratégico en la popularización de la ciencia (Di Carlo, 2015). En particular, Sugimoto et al. (2013) evidenciaron como los videos en línea de las TED talks producen más impacto en el público en general que en la comunidad académica, sobre todo si son protagonizados por científicos, aunque esto no se traduce en un mayor número de citas. Los autores argumentan como los videos online, en particular las TED Talks, pueden ser muy útiles en términos de popularización de la ciencia, pero no para promover el trabajo de científicos e institutos de investigación.

Un obstáculo evidente al uso de los videos en línea en la difusión de la ciencia es la escasa participación de los científicos en la producción de los materiales audiovisuales. Desde el estudio de Sugimoto et. al (2013), que señalaba la escasa presencia de científicos, sobre todo 
mujeres, en la literatura especializada no se registra un incremento significativo del involucramiento y el protagonismo de los científicos en los videos online. Muñoz et al. (2016) ponen de manifiesto una notable profesionalidad de los comunicadores científicos en los vídeos de divulgación en la web, aunque, según el estudio sobre la ciencia y la salud en los medios sociales de Schäfer (2016), no son ellos quienes dominan el escenario mediático en la web por lo que concierne sus temáticas. Dos años después, los científicos y las organizaciones científicas siguen siendo reacios a comunicarse a través de los medios sociales (Allgier, 2018).

\section{La polivalencia de YouTube en la comunicación de la ciencia}

A pesar de la aún limitada participación de los científicos en las nuevas dinámicas comunicativas que involucran los medios y redes sociales, existe un consenso en el reconocer a YouTube como la plataforma libre de videos en línea más utilizada (Berk, 2009) y la más representativa de la nueva cultura audiovisual (Burgess y Green, 2009). En suma, YouTube es empleado para fines distintos y se demuestra polivalente al momento de plantear estrategias de comunicación científica.

\subsection{Versatilidad para la academia}

YouTube es utilizada para la publicación de actividades relacionadas con la investigación, experimentos científicos, conferencias y cursos. Desde hace algunos años existen revistas científicas que permiten cargar archivos de vídeo de los resúmenes los artículos y de material complementario al artículo publicado en la versión digital (Spicer, 2014). Su uso como herramienta para la enseñanza y el aprendizaje es ampliamente reconocido (De Witt et al., 2013) y el acercamiento de la comunidad científica a YouTube no es reciente en cuanto existen 
experiencias que se vinculan a la plataforma desde sus inicios (Kousha, Thelwall y Abdoll, 2012). En la actualidad, las instituciones de educación superior la reconocen como importante medio social y la utilizan dentro de sus estrategias de comunicación.

\subsection{Utilidad en la investigación}

La plataforma de YouTube es utilizada como fuente de información y entorno de investigación. El número de estudios académicos que usan los videos de YouTube como fuente de datos y referencias se incrementa (Soukup, 2014) porque resulta muy útil al momento de explicar los métodos y los procedimientos implementados, y respaldar los argumentos utilizados en las publicaciones. En la observación y estudio de animales, puede reemplazar libros y artículos de revistas por su capacidad de proporcionar nuevos conocimientos en el estudio de especies, especialmente las menos estudiadas (Dylewski et al, 2017). Por su parte, Kousha et al. (2012) analizan qué tipo de vídeos de YouTube se citan formalmente en las publicaciones académicas evaluando las diferencias de uso por ámbito disciplinario. Los resultados muestran un número considerable de videos citados en artículos de revistas de arte y humanidades sobre temas de entretenimiento artístico como la música, la danza, el teatro, la comedia, las películas y las animaciones (p.1715). En cuanto entorno de investigación, YouTube puede ser útil en diseños experimentales controlados para presentar materiales de medios manipulados, como un entorno para estudiar los efectos de la retroalimentación de los pares en diversos contenidos de medios, y para el diseño de cuestionarios (Konijn, Veldhuis y Plaisier, 2015).

\subsection{Eficacia comunicativa}

El potencial de YouTube para la comunicación científica es reconocido por numerosos autores (Allgier, 2018, Erviti y Stengler 2016), su polivalencia y valor estratégico son demostrados por la amplitud de 
usos y fines para los cuales es empleada. La popularidad de la cual goza en la población en línea hace de YouTube un instrumento de alcance mundial capaz de mitigar la falta de información sobre argumentos específicos relevantes para la sociedad, así como influenciar comportamientos y conductas de sus usuarios. Biddinika et al. (2019) analizan sistemáticamente las características de los cien vídeos más vistos en la plataforma sobre cuestiones de energía renovable reconociendo su potencial como herramienta para suplir la falta de información sobre la tecnología de este ámbito científico. En el campo médico, los videos de YouTube son usados para facilitar la comprensión de temas complicados y sensibles para los pacientes, como el cáncer (Sahin, et al., 2019) y las pandemias (Biddinika et al., 2019).

Desde el punto de vista del público, el éxito de YouTube puede ser explicado por la revolución de roles que significó su aparición. Devolvió al público el poder sobre el medio y transformó el video aficionado en un negocio (Burgess y Green, 2009) en el cual la mayor satisfacción del usuario se encuentra en su propia participación tanto en el medio como en el mensaje. Participar en el contenido de un video cientifico permite satisfacer objetivos de aprendizajes (Moll y Nielsen, 2016), produce compromiso y colaboración social (Balakrishnan y Griffiths, 2017), y facilita el relacionamiento de la ciencia con la vida cotidiana (Rosenthal, 2017). En otras palabras, se da a entender un acercamiento del píblico al laboratorio y al campo de estudio estimulando el consumo autónomo y voluntario de contenidos científicos. Sin duda, es necesario tener en cuenta los géneros y formatos de videos más eficaces y la calidad técnica de los productos que se difunden como aspectos fundamentales para el éxito de la comunicación mediante este medio social (Welbourne y Grant, 2015).

\subsection{Interactividad con los públicos de interés}

Antes del cambio de milenio los científicos han iniciado a utilizar los medios electrónicos para comunicarse entre sí, adoptando el nuevo 
sistema de comunicación basado en la colaboración, cooperación y consenso (De Santis-Piras y Jara, 2019). Las redes y medios sociales han impulsado la creación de un nuevo entorno comunicativo para académicos e investigadores a los cuales se presenta la posibilidad de interconectarse e interactuar con pares y ciudadanos, intercambiar conocimientos, impulsar la internacionalización de su trabajo integrándose con organizaciones y actores sociales. YouTube es utilizado para el intercambio de video entre pares, como resultado y producto del proceso investigativo, para la socialización de proyectos en eventos institucionales, y es considerado como una importante herramienta para las relaciones públicas de académicos, centros e instituciones que se dedican a la ciencia.

\section{El papel estratégico del científico/comunicador}

Comprobado que YouTube es un medio funcional a la comunicación de la ciencia y la investigación, y teniendo en cuenta su dinamicidad y el comportamiento de sus usuarios, parece fundamental, desde el punto de vista de la comunicación y planificación estratégica, conocer y analizar el rol que juegan los investigadores como emisores y fuentes de información en el uso que hacen de este servicio para su comunicación. Frente a las múltiples ventajas que ofrece y a su polivalencia comunicativa, se advierte la ausencia de una perspectiva que considere el planteamiento de estrategias y acciones específicas para efectivizar el uso de YouTube en la relación con los públicos de interés. Existen varias buenas razones que motivan la adopción de una perspectiva estratégica para la comunicación científica mediante YouTube:

a. Es necesario captar la atención: la mera disponibilidad de información es insuficiente, y es necesario comprender la forma en que los distintos públicos usan los medios de comunicación y se relacionan en entornos sociales como YouTube. 
b. Mayor actividad produce mayor impacto: la capacidad que tiene YouTube de generar impacto en la comunicación científica depende del grado de actividad de la cuenta/canal o las cuentas/ canales que se administran en la plataforma. La popularidad de una cuenta/canal o un video depende mucho de la gestión de la actualidad, calidad y cantidad de la información que se publica (Susarla, Oh y Tan, 2012; Welbourne y Grant, 2015), el nivel de interacción que se ofrece y su capacidad de adaptación al "sistema multiplataforma, del cual los dispositivos móviles son parte integrante y nodo crucial" (De Santis-Piras y Morales Morante, 2019, p.70).

c. No se trata solo de difusión: El acceso a YouTube se realiza desde distintos medios como motores de búsqueda, sitios web, aplicaciones móviles, blogs y redes sociales, muchas veces usados para difundir y comercializar productos científicos como artículos y libros (Allgier, 2018). El conocimiento es un activo muy importante de una sociedad y como tal debe ser gestionado de manera estratégica. En un entorno mediático sobrecargado de información es imperativo plantear acciones comunicativas eficaces en diferentes escenarios de relación/ confronto que favorezcan la transferencia de conocimiento a la sociedad y la valoración de la ciencia por parte de los distintos públicos.

d. Depende mucho del científico/comunicador: las comunidades de seguidores y usuarios que se forman alrededor de una cuenta o canal en YouTube dependen en gran medida de la capacidad de su creador/gestor de integrarse a las redes sociales de confianza del público, de sus competencias comunicativas, su accesibilidad (Welbourne y Grant, 2015) y, sobre todo, compromiso. Las conexiones y relaciones significativas que un científico y su institución construyen en la web se traducen en visibilidad, posicionamiento, reputación e impacto. Es evidente que la proyección de estos resultados tiene como premisa 
fundamental la presencia de los actores de la ciencia en el entorno virtual.

Este último punto es fundamental y constituye el primer paso necesario para construir una identidad digital, tanto como individuo, grupo o institución, y planificar el uso estratégico de YouTube en la totalidad de conceptos que se han tratado en este apartado: medio social, red social, canal, plataforma digital y servicio. Consideración que genera un cuestionamiento acerca de cómo los científicos plantean su presencia y su identidad digital en la web.

\subsection{Presencia de científicos en YouTube: el caso de los autores de Publicidad y Relaciones Públicas}

En el ámbito de la Publicidad y las Relaciones Públicas, los científicos no parecen considerar YouTube como una herramienta estratégica para sus actividades de comunicación científica. De una muestra de autores científicos seleccionada en base al impacto de su publicación más citada por pares, se ha analizado la presencia en la plataforma de YouTube como punto de partida para entender el valor estratégico que esta comunidad le atribuye como herramienta de comunicación. La muestra se compone del total de los autores de los cinco artículos más citados en Scopus en el periodo entre 2009 y 2018 de las revistas científicas que tratan explícitamente de Publicidad (3) y Relaciones Públicas (3) ubicada en el Cuartil 1 (Q1) del Scimago Journal Ranking 2018. Las revistas seleccionadas en el ámbito de Publicidad son Journal of Advertising, International Journal of Advertising y Journal of Advertising Research; para Relaciones Públicas las revistas resultan Public Relation Review, Journal of Public Relations Research y Public Relations Inquiry. Se usa Scopus por el reconocido valor científico como base de datos y por su mayor amplitud respecto a Web of Science, otra institución de referencia en materia, en consideración de la comprobada superposición entre ambas bases de datos (Kousha et al., 2012). Se obtiene una muestra final de 59 autores de los cuales 32 en el 
grupo de Publicidad y 27 en el grupo de Relaciones Públicas con una distribución que no presenta diferencias significativas ( $p$ o.852) entre grupos y por concepto de sexo. La muestra final ha sido depurada de aquellos autores (16) que no han seguido la carrera académica y/o de investigadores luego de su publicación o la han abandonado antes de 2019. Del total de autores solo el 11,9\% dispone de una cuenta activa en YouTube, y de estos solo el 3,4\% la utiliza para actividades de comunicación científica (Tabla 1).

\section{Tabla 1: Autores de Publicidad y Relaciones Públicas con cuenta activa en YouTube}

\begin{tabular}{lrcc} 
& \multicolumn{3}{c}{ YouTube } \\
\cline { 2 - 4 } \multicolumn{1}{c}{ AREA } & Ausente & No cientifico & Cientifico \\
\hline Publicidad & $90.6 \%$ & $3.1 \%$ & $6.3 \%$ \\
Relaciones Publicas & $85.2 \%$ & $14.8 \%$ & $0.0 \%$ \\
Total & $88.1 \%$ & $8.5 \%$ & $3.4 \%$ \\
\hline
\end{tabular}

La presencia marginal de los autores de la muestra en YouTube resulta más relevante si se considera que son académicos y profesionales vinculados con áreas científicas tales como: Publicidad y Relaciones Públicas (27,1\%), Comunicación (23,7\%), Marketing (20,3\%), Negocios (10,2\%), Comunicación Estratégica (6,8\%) y Periodismo $(5,1 \%)$ entre las que más se destacan. Por otro lado, se ha realizado un análisis de la presencia de los autores en los videos en línea de YouTube compartidos por otros usuarios individuales o institucionales. La clasificación considera cuatro tipologías de contenidos: conferencias académicas, entrevistas en medios de comunicación, material didáctico y contenido no académico. En la última categoría se incluye todo lo que no está relacionado con las tres primeras (Tabla 2). Es evidente como las conferencias $(56,3 \%)$ y las entrevistas $(55,8 \%)$ son 
el formato con el cual más de la mitad de los autores se encuentra en YouTube en videos realizados por terceros. En particular, se trata de instituciones de educación superior y asociaciones organizadoras de congresos y simposios en el caso de las conferencias, y los medios de comunicación tradicionales y digitales para las entrevistas. Casi un tercio de los autores está en YouTube con videos que no conciernen la actividad de científico, más bien su vida privada, y es el caso de aquellos que disponen de una cuenta en la plataforma. Hay que señalar que ninguno de estos videos es actual y las cuentas personales aparentan estado de abandono.

\section{Tabla 2: Presencia de los autores en los videos de YouTube}

Tabla 2: Presencia de los autores en los videos de YouTube

\begin{tabular}{|c|c|c|c|c|}
\hline \multirow[b]{2}{*}{ Area cientifica } & \multicolumn{4}{|c|}{ Tipo de Video } \\
\hline & Conferencia & Fntrevista & $\begin{array}{l}\text { Material } \\
\text { didactico }\end{array}$ & $\begin{array}{c}\text { No } \\
\text { académico }\end{array}$ \\
\hline Publicidad & 15,696 & $18,8 \%$ & $6,3 \%$ & 9,496 \\
\hline Relaciones Pablicas & 40,796 & $37 \%$ & 11,196 & 22,296 \\
\hline Total & 56,396 & $55.8 \%$ & $17,4 \% 6$ & 31.646 \\
\hline
\end{tabular}

Finalmente, es necesario señalar que los autores son científicos, académicos y/o profesionales de diferentes países con una neta prevalencia de Estados Unidos (55,9\%), además de Corea del Sur (10,2\%), Australia (8,5\%), Alemania, Países Bajos y Reino Unido (5,1\%), Suecia y Hong Kong (3,4\%), Emiratos Árabes y Canadá (1,7\%). Los resultados son parte de una investigación que uno de los autores está realizando en la Universitat Autónoma de Barcelona sobre la comunicación estratégica de la ciencia en Internet, un proyecto en curso de obra, por lo cual son parciales y se limitan a la descripción del fenómeno. El estudio se vuelve consistente al momento de analizar la presencia y el 
uso de otras plataformas digitales como sitios web y redes sociales. De todas maneras, ofrecen una visión suficientemente clara y permiten inferir sobre el uso estratégico de los medios digitales por parte de los científicos de los ámbitos disciplinarios involucrados en el estudio.

\section{Conclusiones}

Lasiniciativas enlaweb puestas en marchaporinstitucionesycientíficos de todo el mundo para comunicar la ciencia con mayor eficacia han sido diversas (Allgier, 2018), sobre todo a través de video en línea y mediante el uso de YouTube. Sin duda, la presencia y participación de los científicos es todavía escasa. En general, los científicos que se exponen y protagonizan iniciativas personales o proyectos institucionales fuera de los canales y plataformas tradicionales de comunicación científica aún siguen siendo pocos, perdiendo así una gran oportunidad para incrementar visibilidad e impacto de la labor científica que desarrollan individuos e instituciones.

Los videos de YouTube son usados como referencias en las publicaciones académicas, lo que puede favorecer el interés y el acceso al paper o libro vinculado con el video, aunque estas no se traduzcan necesariamente en citas para los autores. El uso de YouTube como herramienta estratégica puede ser particularmente útil a las realidades asociativas e institucionales como grupos, core, centros e institutos de investigación, con el fin de mejorar su comunicación con los públicos de interés, optimizar la difusión del conocimiento generado e incrementar el impacto de sus publicaciones.

Finalmente, cabe señalar la oportunidad para las instituciones, entes públicos y privados responsables del fomento de la investigación y la comunicación científica, de apoyar con recursos e incentivos el uso de YouTube y otros servicios digitales que puedan significar un salto de calidad en término de comunicación de la ciencia y relación de sus actores con los públicos de interés y la sociedad. 


\section{Referencias}

Alexa (2020). The top 500 sites on the web. Recuperado el 16 de febrero 2020 de https://www.alexa.com/topsites

Allgaier, J. (2020). Science and Medicine on YouTube. Second International Handbook of Internet Research, 7-27. doi: 10.1007/978-94-024-1555-1_1

Armstrong, M. (11 de marzo de 2019). YouTube is Responsible for $37 \%$ of All Mobile Internet Traffic. Statista. Recuperado de https://www.bit.ly//2PSKffd

Balakrishnan, J., y Griffiths, M. D. (2017). Social media addiction: What is the role of content in YouTube? Journal of Behavioral Addictions, 6(3), 364-377.

Berk, R. A. (2009). Multimedia teaching with video clips: TV, movies, YouTube, and $\mathrm{mtvU}$ in the college classroom. International Journal of Technology in Teaching and Learning, 5(1), 1-21.

Biddinika, M. K., Syamsiro, M., Novianti, S., Nakhshiniev, B., Aziz, M., y Takahashi, F. (2019). Dissemination of technology information through YouTube: A case of renewable energy technology. Telkomnika (Telecommunication Computing Electronics and Control), 17(3), 1526-1538. doi: 10.12928/TELKOMNIKA.V17I3.10124

Brossard, D. (2013). New media landscapes and the science information consumer. Proceedings of the National Academy of Sciences, 110(Supplement 3), 14096 LP - 14101. doi: 10.1073/pnas.1212744110

Burgess, J. , y Green, J. (2009). YouTube: Online video and participatory culture. Malden, EEUU: Polity Press.

Datareportal (2019). digital 2019: global digital overview. Recuperado de https:// www.bit.ly/2IqjMBr

de Lara González, A., y García-Avilés, J. A. (2019). Estudio de la calidad del vídeo online en la comunicación de la ciencia. Perspectivas de la comunicación, 12(1), 185-207. doi: 10.4067/So718-48672019000100185

De Santis-Piras, A., y Jara Cobos, R.V. (2019). Comunicación de la investigación en la web: las plantas medicinales y la FarmaciaAncestral.ups.edu.ec. En A. De Santis-Piras y A. Verdugo-Sanchez (Eds.), Salud, interculturalidad y Buen Vivir: Respeto a la diversidad y mutuo beneficio en el intercambio de saberes $y$ experiencias (pp. 131-147). Quito, Ecuador: Abya-Yala. 
De Santis-Piras A., y Morales Morante, L. (2019). Dispositivos móviles y las multiplataforma de interacción lúdica. En A. Torres Toukumidis, L. Romero-Rodríguez y J. Salgado Guerrero (Eds.), Juegos y Sociedad: desde la interacción a la inmersión para el cambio social (pp. 69-78). Ciudad de México: McGraw Hill.

DeWitt, D., Alias, N., Siraj, S., Yusaini, M., Ayob, J., e Ishak, R. (2013). The Potential of YouTube for Teaching and Learning in the Performing Arts. Procedia - Social and Behavioral Sciences, 103, 1118 - 1126. doi: 10.1016/j. sbspro.2013.10.439

Díaz Pérez, M., de Moya Anegón, F., y Carrillo-Calvet, H. (2018). Técnicas para la visualización de dominios científicos y tecnológicos. Investigación Bibliotecológica: archivonomía, bibliotecología e información, o(nesp1), 17-42. doi: 10.22201/iibi.24488321xe.2017.nesp1.57884

Di Carlo, G. (2015). Pathos as a communicative strategy for online knowledge dissemination: The case of TED talks. 3L: Language, Linguistics, Literature, 21(1), 23-34. doi: 10.17576/3L-2015-2101-03

Dylewski, Ł., Mikula, P., Tryjanowski, P., Morelli, F., y Yosef, R. (2017). Social media and scientific research are complementary-YouTube and shrikes as a case study. The Science of Nature, 104(48). doi: 10.1007/soo114-017-1470-8

Fundación Española para la Ciencia y la Tecnología Fecyt, (2018). IX Epscyt 2018 informe de resultados (9). Recuperado de https://bit.ly/2v1TFh4

Francés, M., y Peris, A. (2018). Rigour in Online Science Videos: An Initial Approach. En B. León y M. Bourk (Eds.), Communicating Science and Technology Through Online Video (64-76). New York: Routledge. doi: 10.4324/9781351054584

Gheorghiu, A. I., Callan, M. J., y Skylark, W. J. (2020). A Thin Slice of Science Communication: Are People's Evaluations of TED Talks Predicted by Superficial Impressions of the Speakers? Social Psychological and Personality Science, 11(1), 117-125. doi: 10.1177/1948550618810896

Komada, M., Takao, K. y Nakanishi K., y Miyakawa, T. (2009). The potential benefit of JoVE, an online video journal for science. Journal of Information Processing and Management, 52(2), 69-76. doi: 10.1241/johokanri.52.69 
Kousha, K., Thelwall, M., y Abdoli, M. (2012). The role of online videos in research communication: A content analysis of YouTube videos cited in academic publications. Journal of the American Society for Information Science and Technology, 63(9), 1710-1727. doi: 10.1002/asi.22717

Konijn, E., Veldhuis, J., y Plaisier, X.S. (2013). YouTube as a research tool: three approaches. Cyberpsychology, Behavior, and Social Networking, 16(9), 695701. doi: 10.1089/cyber.2012.0357

JoVE, (2020). Accelerating Scientific Research and Education. Recuperado el 16 de febrero 2020 de https://www.jove.com/about/

Laakso, M., Lindman, J., Shen, C., Nyman, L., y Björk, B. C. (2017). Research output availability on academic social networks: Implications for stakeholders in academic publishing. Electronic Markets, 27(2), 125-133. doi: 10.1007/ s12525-016-0242-1

León, B., y Bourk, M. (Eds.) (2018). Communicating Science and Technology Through Online Video. New York: Routledge. doi: 10.4324/9781351054584.

Moll, R., y Nielsen, W. (2016). Development and validation of a social media and science learning survey. International Journal of Science Education, Particle B, 14-30. doi:10.1080/21548455.2016.1161255

Muñoz, M. J., Czurda, K., y Robertson-von Trotha, C. Y. (2016). Typologies of the popular science web video. Journal of Science Communication, 15(04), A02. doi: 10.22323/2.15040202

Oh, S., Baek, H., y Ahn, J. (2017). Predictive value of video-sharing behavior: Sharing of movie trailers and box-office revenue. Internet Research, 27(3), 691-708. doi: 10.1108/IntR-01-2016-0005

Pan, X., Yan, E., y Hua, W. (2015). Science communication and dissemination in different cultures: An analysis of the audience for TED videos in China and abroad. Journal of the Association for Information Science and Technology, 67(6), 1473-1486. doi:10.1002/asi.2346

Robinson-García, N., Repiso, R., y Torres-Salinas, D. (2018). Perspectiva y retos de los profesionales de la evaluación científica y la bibliometría. El Profesional de La Información, 27(3), 461-466. doi: doi.org/10.3145/epi.2018.may.o1 
Rosenthal, S. (2017). Motivations to seek science videos on YouTube: freechoice learning in a connected society. International Journal of Science Education, Part B, doi:10.1080/21548455.2017.1371357

Sahin, A. N., Sahin, A. S., Schwenter, F., y Sebajang, H., (2019). YouTube Videos as a Source of Information on Colorectal Cancer: What Do Our Patients Learn? Journal of Cancer Education, 34(6), 1160-1166. doi: 10.1007/s13187-018-1422-9 Schäfer, M. (2016). Wissenschaftskommunikation online. En H. Bonfadelli, B. Fähnrich, C. Lüthje, J. Milde, M. Rhomberg, y M.S. Schäfer (Eds.), Forschungsfeld Wissenschaftskommunikation (275-293). Heidelberg, Alemania: Springer.

Sonne, S. C., Andrews, J. O., Gentilin, S. M., Oppenheimer S., Obeid, J., Brady, K.,...Magruder, K. (2013). Development and pilot testing of a video-assisted informed consent process. Contemporary Clinical Trials, 36(1), 25-31. doi: 10.1016/j.cct.2013.05.011

Soukup, P. A. (2014). Looking at, with, and through YouTube. Communication Research Trends, 33(3), 3-34.

Spicer, S. (2014). Exploring video abstracts in science journals: an overview and case study. Journal of Librarianship and Scholarly Communication, 2(2), eP1110. doi: 10.7710/2162-3309.1110

Statista (2020). Global social networks ranked by number of users 2020. Recuperado de https://www.bit.ly/2VT9G3S

Stern, D. (2013). The Future of Peer-Reviewed Scientific Video Journals. Online Search, 37(3), 28-32.

Sugimoto, C. R., Thelwall, M., Larivière, V., Tsou, A., Mongeon, P., y Macaluso, B. (2013). Scientists Popularizing Science: Characteristics and Impact of TED Talk Presenters. PLoS ONE, 8(4), e62403. doi:10.1371/journal.pone.0062403 Susarla, A., Oh, J. H., y Tan, Y. (2012). Social networks and the diffusion of user-generated content: Evidence from YouTube. Information Systems Research, 23(1), 23-41. doi: 10.1287/isre.1100.0339

Tseng, C. H., y Huang, T. L. (2016). Internet advertising video facilitating health communication: Narrative and emotional perspectives. Internet Research, 26(1), 236-26. doi: 10.1108/IntR-09-2014-0217 
Vázquez-Cano, E. (2013). El videoartículo: nuevo formato de divulgación en revistas científicas y su integración en MOOCs. Comunicar: Revista Científica de Comunicación y Educación, 21(41), 83-91. doi: 10.3916/C41-2013-08

Verjovsky, M., y Jurberg, C. (2019). Spreading Ideas: TED Talks' Role in Cancer Communication and Public Engagement. Journal of Cancer Education. doi: 10.1007/s13187-019-01583-6

Welbourne, D. J., y Grant, W. J. (2015). Science communication on YouTube: Factors that affect channel and video popularity. Public Understanding of Science, 25(6), 706-718. doi:10.1177/0963662515572068 